\title{
Optimal discrete hedging in the Heston Stochastic Volatility Model
}

\author{
Toby Daglish*and Chris Neely ${ }^{\dagger}$
}

August 23, 2007

\begin{abstract}
We present a closed form solution for the optimal hedging strategy, in discrete time, of an option whose underlying security follows the Heston Stochastic Volatility process. Our Monte Carlo simulations indicate that this significantly improves hedging performance at weekly and longer hedging intervals, when compared to continuous time hedging procedures.
\end{abstract}

JEL Classification: G11: Portfolio Choice, G12: Asset Pricing.

Prices for options are directly observable in the market, while the sensitivities of these prices to changes in underlying state variables are not. As such, an important application of option pricing models is the calculation of these hedge statistics, or "Greek letters".

Not surprisingly, comparisons of hedging performance have become an important part of the empirical options pricing literature. Many empirical studies (such as Bakshi, Cao, and Chen (1997), Hutchinson, Lo, and Poggio (1994) and Dumas, Fleming, and Whaley (1998)) compare models on the basis of their ability to construct tracking portfolios.

For both empirical studies, and real world hedging applications, one must assume that either the data are discrete (in the former case) or that trading opportunities are discrete (in the latter case) due to transactions costs. Wilmott (1994) presents optimal discrete time hedging formulae for the Black-Scholes framework.

Stochastic volatility was an important extension of the Black-Scholes model, especially the Heston (1993) model. Stochastic volatility has become an established feature of both the empirical and theoretical options pricing literature. Branger and Schlag (2004), for example, attempt to quantify the size of tracking error for discrete option hedging in the presence of stochastic volatility. They conclude that discreteness error renders the delta hedging error an unreliable guide to volatility risk premia.

This paper tackles the stochastic volatility discrete hedging problem, using a framework similar to Wilmott (1994). We are thus able to calculate exact formulae for the optimal hedging strategies in discrete time for an option whose underlying security is subject to Stochastic Volatility. These results will be of use to both empiricists seeking to accurately measure the hedging benefit of the Heston model, and to practitioners who are seeking to use the model for hedging.

\footnotetext{
*Victoria University of Wellington. E-mail: Toby.Daglish@vuw.ac.nz.

${ }^{\dagger}$ Federal Reserve Bank of St. Louis. E-mail: Christopher.J.Neely@stls.frb.org.
} 
The remainder of the paper is as follows: section 1 contains our main result: the optimal hedging strategy in discrete time for an option subject to stochastic volatility; section 2 presents some simulation results demonstrating the magnitude of this effect; section 3 concludes.

\section{Optimal Hedging}

This paper derives the variance minimising strategy for an agent who has a position in one derivative, and has access to a second derivative (written on the same underlying security) as well as the underlying security with which to hedge this position. Note that these results are not specific to European options, or even to options in general. Any security which obeys the Heston PDE may be hedged (or used as a hedge security) in this analysis.

Proposition 1 Consider an agent holding a portfolio consisting of two options (holdings $\pi_{1}$ and $\pi_{2}$ ) and the underlying security $\left(\pi_{0}\right)$. If we denote the prices of the options by $f_{i}$, the underlying asset's price as $s$ and its dividend rate as $q$, then over an interval of time $(\Delta t)$ the portfolio changes in value according to:

$$
\Delta W=\pi_{1} \Delta f_{1}+\pi_{2} \Delta f_{2}+\pi_{0}(\Delta s+q s \Delta t)
$$

where the evolution of $s$ is governed by:

$$
\begin{aligned}
d s & =\mu s d t+\sqrt{v} s d z_{1} \\
d v & =(\alpha-\beta v) d t+\sigma_{v} \sqrt{v} d z_{2}
\end{aligned}
$$

where $d z_{1}$ and $d z_{2}$ are two Brownian motions with correlation $\rho$. Holding $\pi_{1}$ fixed, the optimal choice of $\pi_{0}$ and $\pi_{2}$, to minimise the variance of $\Delta W$, is given by:

$$
\begin{aligned}
& \pi_{0}=-f_{2 s} \pi_{2}-\pi_{1}\left[f_{1 s}+\left(\frac{A_{10} f_{2 v}+A_{11} \rho v f_{1 v}}{4 s \sigma_{v} f_{2 v}^{2}\left(1-\rho^{2}\right)}\right) \Delta t\right] \\
& \pi_{2}=-\frac{\pi_{1}}{f_{2 v}}\left[f_{1 v}+\left(\frac{A_{20} f_{2 v}+2 A_{21} v f_{1 v}}{4 \sigma_{v}^{2} f_{2 v}^{2}\left(1-\rho^{2}\right)}\right) \Delta t\right]
\end{aligned}
$$

where the constants $A_{10}, A_{11}, A_{20}$ and $A_{21}$ are given by:

$$
\begin{aligned}
A_{10}= & s^{2} \sigma_{v}\left(f_{2 s s} f_{1 v}-f_{2 v} f_{1 s s}\right)\left[4\left(\rho^{2}-1\right)(\mu+q-r)-2 v+\sigma_{v} \rho\left(\rho^{2}+1\right)\right] \\
& +\rho\left[\left(\sigma_{v}^{4}-2 \rho \sigma_{v}^{3} v\right)\left(f_{2 v v} f_{1 v}-f_{1 v v} f_{2 v}\right)+\left(2 \rho s \sigma_{v}^{3}-4 \sigma_{v}^{2} s v\right)\left(f_{2 s v} f_{1 v}-f_{1 s v} f_{2 v}\right)\right. \\
& -2 \sigma_{v}^{4} v f_{2 v v} f_{1 v v}-2 \sigma_{v}^{2} \rho^{2} v s^{2}\left(f_{2 s s} f_{1 v v}+f_{1 s s} f_{2 v v}\right)-2 v s^{4} f_{2 s s} f_{1 s s} \\
& \left.-4 s \sigma_{v}^{3} \rho v\left(f_{2 s v} f_{1 v v}+f_{1 s v} f_{2 v v}\right)-4 \rho v s^{3} \sigma_{v}\left(f_{2 s s} f_{1 s v}+f_{1 s s} f_{2 s v}\right)-4 \sigma_{v}^{2}\left(1+\rho^{2}\right) v s^{2} f_{2 s v} f_{1 s v}\right] \\
A_{11}= & 2 \sigma_{v}^{4} f_{2 v v}^{2}+4 \sigma_{v}^{2}\left(1+\rho^{2}\right) s^{2} f_{2 s v}^{2}+8 s \sigma_{v}^{3} \rho f_{2 s v} f_{2 v v} \\
& +2 s^{4} f_{2 s s}^{2}+4 \sigma_{v}^{2} \rho^{2} s^{2} f_{2 s s} f_{2 v v}+8 \rho s^{3} \sigma_{v} f_{2 s s} f_{2 s v},
\end{aligned}
$$


and

$$
\begin{aligned}
A_{20}= & \left(f_{2 s v} f_{1 v}-f_{2 v} f_{1 s v}\right) s \sigma_{v}^{2}\left(4\left(\rho^{2}-1\right)(\mu+q-r)+2 \rho^{2}\left(2 v-\rho \sigma_{v}\right)\right) \\
& +\left(\sigma_{v}^{4}-2 \sigma_{v}^{3} \rho^{3} v\right)\left(f_{2 v} f_{1 v v}-f_{1 v} f_{2 v v}\right)+\left(3 s^{2} \sigma_{v}^{2} \rho^{2}-2 \sigma_{v} \rho v s^{2}-s^{2} \sigma_{v}^{2}\right)\left(f_{2 v} f_{1 s s}-f_{1 v} f_{2 s s}\right) \\
& +2 v s^{4} f_{2 s s} f_{1 s s}+2 v \sigma_{v}^{4} f_{2 v v} f_{1 v v}+4 v \sigma_{v}^{2} s^{2}\left(1+\rho^{2}\right) f_{2 s v} f_{1 s v} \\
& +4 v s \sigma_{v}^{3} \rho\left(f_{2 v v} f_{1 s v}+f_{2 s v} f_{1 v v}\right) \\
& +2 v \sigma_{v}^{2} s^{2} \rho^{2}\left(f_{2 s s} f_{1 v v}+f_{1 s s} f_{2 v v}\right)+4 v s^{3} \rho \sigma_{v}\left(f_{2 s s} f_{1 s v}+f_{2 s v} f_{1 s s}\right) \\
A_{21}= & -\left(2 s^{2} \sigma_{v}^{2}\left(1+\rho^{2}\right) f_{2 s v}^{2}+4 s \sigma_{v}^{3} \rho f_{2 s v} f_{2 v v}+s^{4} f_{2 s s}^{2}\right. \\
& \left.+2 s^{2} \sigma_{v}^{2} \rho^{2} f_{2 s s} f_{2 v v}+4 s^{3} \rho \sigma_{v} f_{2 s s} f_{2 s v}+\sigma_{v}^{4} f_{2 v v}^{2}\right) .
\end{aligned}
$$

Proof: See Appendix A

This formula relates the optimal hedging strategy to the second order moments of the two option prices. Note that if options one and two are identical (so that $f_{1 s}=f_{2 s}, f_{1 v}=f_{2 v}$, etc) then the strategy collapses to:

$$
\pi_{0}=0 \quad \pi_{2}=-\pi_{1},
$$

or in other words: simply take an exactly offsetting position in the identical option. Some other special cases of the result are also worth noting.

First, consider the delta hedging case, in which $\pi_{2}$ equals zero. In this case we find:

Proposition 2 Suppose we have the framework from 1 with the exception that $\pi_{2}=0$ (the agent cannot use the second option), then the optimal position in the underlying security is given by:

$$
\pi_{0}=-\pi_{1}\left(\frac{\sigma_{v} \rho f_{1 v}}{s}+f_{1 s}+\frac{A_{3}}{2 s} \Delta t\right)
$$

where

$$
\begin{aligned}
A_{3}= & -\rho^{2} s \sigma_{v}^{2} f_{1 s v}+\rho^{2} \sigma_{v}^{2} v f_{1 v v}+2 \rho \sigma_{v} s f_{1 s v}(\mu+v-r+q)+2 s^{2} f_{1 s s}\left(\mu+q+\frac{v}{2}-r-\frac{\sigma_{v} \rho}{2}\right) \\
& -2 \sigma_{v} \rho f_{1 v}\left(\beta+q-r+\frac{v}{2}-\frac{\rho \sigma_{v}}{4}\right) .
\end{aligned}
$$

Proof: See Appendix B.

If volatility is uncorrelated to the stock process $(\rho=0)$ or deterministic $\left(\sigma_{v}=0\right)$ the the solution, $\pi_{0}$, reduces to the case described by Wilmott:

$$
\pi_{0}=-\pi_{1}\left(f_{1 s}+s f_{1 s s}\left(\mu+q+\frac{v}{2}-r\right) \Delta t\right) .
$$

Note also that for the purely delta hedging case (i.e. $p i_{2}=0$ ) letting $\Delta t$ go to zero (continuous hedging) means that the optimal solution becomes:

$$
\pi_{0}=-\pi_{1}\left(\frac{\sigma_{v} \rho f_{1 v}}{s}+f_{1 s}\right) .
$$


That is, an optimal discrete hedging strategy allows for some vega hedging through the use of the stock alone (provided $\rho \neq 0$. Similarly for the full two asset hedge, we find that (for $\Delta t=0$ ):

$$
\begin{aligned}
\pi_{2} & =-\pi_{1} \frac{f_{1 v}}{f_{2 v}} \\
\pi_{0} & =\pi_{1} \frac{f_{2 s} f_{1 v}-f_{1 s} f_{f_{2} v}}{f_{2 v}} \\
& =-\pi_{1} f_{1} s-\pi_{2} f_{2} s
\end{aligned}
$$

so that the optimal behaviour is to vega hedge with the second option, and delta hedge the combined risk of the two positions.

If we consider the case where volatility is constant $\left(\sigma_{v}=0\right)$ and both assets are available, we obtain:

Proposition 3 Suppose that we have the framework of Proposition 1, with the further assumption that $\sigma_{v}=0$. Then the optimal hedge is given by:

$$
\begin{aligned}
& \pi_{2}=-\pi_{1} \frac{f_{1 s s}}{f_{2 s s}} \\
& \pi_{0}=-\pi_{2} f_{2 s}-\pi_{1} f_{1 s}
\end{aligned}
$$

Proof: See Appendix C.

This result shows that the investor should gamma hedge with the second option, and then delta hedge with the stock: an appropriate strategy if volatility were constant. ${ }^{1}$

Lastly, we can consider the case stock prices and volatility are uncorrelated, that is, where $\rho=0$. Here we obtain:

Proposition 4 Suppose that we have the framework of Proposition 1, with the further assumption that $\rho=0$. Then the optimal hedge is given by:

$$
\begin{aligned}
& \pi_{0}=-f_{2 s} \pi_{2}-\pi_{1}\left(f_{1 s}-s \frac{f_{2 s s} f_{1 v}-f_{2 v} f_{1 s s}}{f_{2 v}}\left[\mu+q-r-\frac{v}{2}\right] \Delta t\right) \\
& \pi_{0}=-\frac{\pi_{1}}{f_{2 v}}\left[f_{1 v}+\left(\frac{A_{40} f_{2 v}+2 v f_{1 v} A_{41}}{4 \sigma_{v}^{2} f_{2 v}^{2}}\right) \Delta t\right]
\end{aligned}
$$

where

$$
\begin{aligned}
A_{40}= & \left(f_{2 v} f_{1 s v}-f_{2 s v} f_{1 v}\right) s \sigma_{v}^{2}[4(\mu+q-r)] \\
& +\sigma_{v}^{4}\left(f_{2 v} f_{1 v v}-f_{1 v} f_{2 v v}\right)-s^{2} \sigma_{v}^{2}\left(f_{2 v} f_{1 s s}-f_{1 v} f_{2 s s}\right) \\
A_{41}= & 2 s^{2} \sigma_{v}^{2}\left(f_{2 v} f_{2 s v} f_{1 s v}-f_{1 v} f_{2 s v}^{2}\right)+s^{4}\left(f_{2 v} f_{1 s s} f_{2 s s}-f_{1 v} f_{2 s s}^{2}\right) \\
& +\sigma_{v}^{4}\left(f_{2 v} f_{1 v v} f_{2 v v}-f_{1 v} f_{2 v v}^{2}\right) .
\end{aligned}
$$

Proof: This follows from substituting $\rho=0$ into Proposition 1.

\footnotetext{
${ }^{1}$ Note that here there is no term of $O(\Delta t)$ since the gamma hedge will reduce tracking error to order $O\left(\Delta t^{2}\right)$. A potential avenue for future research would be to include terms of $O\left(\Delta t^{2}\right)$, which would shed some light on the efficacy of gamma hedging in discrete time.
} 


\begin{tabular}{lr}
\hline Parameter & Value \\
\hline$\alpha$ & 0.08 \\
$\beta$ & 2 \\
$\sigma_{v}$ & 0.2 \\
$\rho$ & 0.1 \\
$r$ & 0.02 \\
$q$ & 0.03 \\
$\mu$ & -0.01 \\
\hline
\end{tabular}

Table 1: Parameters for the Monte Carlo simulation. Note that these parameters imply a long term volatility of $\sqrt{0.08 / 2}=0.2$.

\section{Tracking Errors}

To test the performance of our hedging rule, we simulate data from a Heston model with parameters given in table 1.

We consider an initial stock price of $\$ 50$, and two one year European call options, one with a strike price of $\$ 49$ (to be hedged) and the other with strike price of $\$ 47.5$ (to be used as a hedge instrument). Following our previous notation $\pi_{1}=-1$ (our objective is to hedge a short position). We simulate data using the standard Euler approximation, with time step of 0.0005 .

We then consider four different strategies for hedging the option with strike price \$49: a conventional delta hedge, a conventional delta and vega hedge (using the option with strike price $\$ 47.5$ ), the Wilmott discrete time delta hedge, our discrete delta hedge (from Proposition 2) and our discrete time delta/vega hedge (from Proposition 1). For the continuous delta hedges, we consider the hedging strategy implied by the Heston model and the continuous time BS hedging strategy calculated using the option's BS IV. Similarly, for the Wilmott hedge, we consider both using greeks calculated from the Heston model, and also using Black-Scholes greeks (again inferred from the option's implied volatility). We consider various sizes of discrete heding intervals $(\Delta t)$ ranging from 0.005 (approximately one day) to 0.1 (which is approximately a monthly hedging interval). In each case, we calculate two statistics, first a standard deviation (Table 2) calculated as:

$$
\text { S.D. }=\sqrt{\frac{1}{J} \sum_{j=1}^{J}\left[\sum_{t=1}^{T} \epsilon_{(\tau-1) \Delta t, \tau \Delta t}\right]^{2}-\left[\frac{1}{J} \sum_{j=1}^{J} \sum_{t=1}^{T} \epsilon_{(\tau-1) \Delta t, \tau \Delta t}\right]^{2}}
$$

and a cumulative tracking error (Table 3), being defined as:

$$
\text { T.E. }=\frac{1}{J} \sum_{j=1}^{J} \sum_{\tau=1}^{T}\left(\epsilon_{(\tau-1) \Delta t, \tau \Delta t}\right)^{2} .
$$

In both cases, $J$ is the total number of simulations (in our case 1000) $T \Delta t$ is the period over which the cumulative tracking error is calculated, and

$$
\epsilon_{s, t}=\pi_{0}(s)(S(t)-S(s))+\pi_{0}(s) \int_{s}^{t} q S(\tau) d \tau+\pi_{2}(s)\left(f_{2}(t)-f_{2}(s)\right)-\left(f_{1}(t)-f_{1}(s)\right)
$$


represents the profit (or loss) due to a particular hedge portfolio held in conjunction with a short position in the option to be hedged. We approximate the integral as:

$$
\int_{s}^{t} q S(\tau) d \tau \approx \sum_{j=1}^{(t-s) / 0.005} q S(s+0.005 j) .
$$

Vega hedging, whether of our adjusted form or the continuous method, can lead to very poor results if used when the hedging option has very low vega. In these situations, calculating the hedge position requires dividing by a very small number, which amplifies any approximation errors in the calculation of vega for either option. Hence, throughout these simulation results, for cases where $\frac{\partial f}{\partial v}<0.1$ for either option, we abandon vega hedging, and simply delta hedge. In the case of the unadjusted hedge, we use continuous time delta hedging (with the Heston delta) while in the case of the discrete adjusted hedge, we use the Proposition 2 correction.

Table 2 examines the standard deviation metric. The columns of the table represent our seven possible strategies: the Proposition 1 hedge (DN1) a continuous time delta/vega hedge (DV) a hedge based on Proposition 2 (DN2) a continuous time delta hedge (D) and a discrete hedge based on Wilmott's result(W). For the latter four hedges, we consider using either the Heston sensitivities, or Black-Scholes sensitivities. We find that for daily $(\Delta t=0.005)$ or bi-daily $(\Delta t=0.01)$ hedging intervals, DN1 gives similar performance to DV. However, with a weekly hedging interval $(\Delta t=0.025)$ it gives a $28 \%$ performance improvement. This grows to a $41 \%$ improvement at the fortnightly interval $(\Delta t=0.05)$ and a $50 \%$ improvement at monthly hedging $(\Delta t=0.1)$.

Of the strategies which do not use a second option to hedge vega (the proposition 2 hedge, the continuous delta hedge and the Wilmott hedge) we find that the conventional delta hedge using a delta calculated from the Heston model (D) is the best performer. Note, however, that all of these approaches suffer from worsening performance as $\Delta t$ increases. Indeed, for the monthly hedging case, we find that delta hedging of any kind leads to a greater portfolio variation than simply holding an unhedged short position in the option. Even the continuous vega hedge performs poorly in this case, yielding only a 17\% improvement over not hedging, compared to the $41 \%$ improvement of the proposition 1 hedge. At a one month hedging interval, the proposition 1 hedge performance is comparable to the continuous delta-vega hedge at a fortnightly rebalancing interval.

The Tracking-Error metric penalises any deviation of the hedge portfolio from the option's price movements, rather than only comparing the overall profit/loss over an interval. The results here are qualitatively similar to the standard deviation results. At a weekly interval, the DN method (proposition 1) shows a $36 \%$ reduction in error compared to DV (continuous delta/vega) hedging. This grows to a $59 \%$ improvement at the monthly interval.

In this case, we note that the delta hedging strategies (D,DN2 and W) do not degrade as rapidly, and are still much superior to not hedging even at a monthly rebalancing interval. This difference suggests that delta hedging at longer rebalancing intervals may add some value over the earlier part of the option, where the option's price is not changing as rapidly as at maturity time.

According to the tracking error metric, including another option when hedging provides a substantial improvement at any rebalancing interval. Our proposition 1 hedge, with monthly rebalancing, gives comparable performance to a bi-daily rebalanced delta-hedged portfolio. 


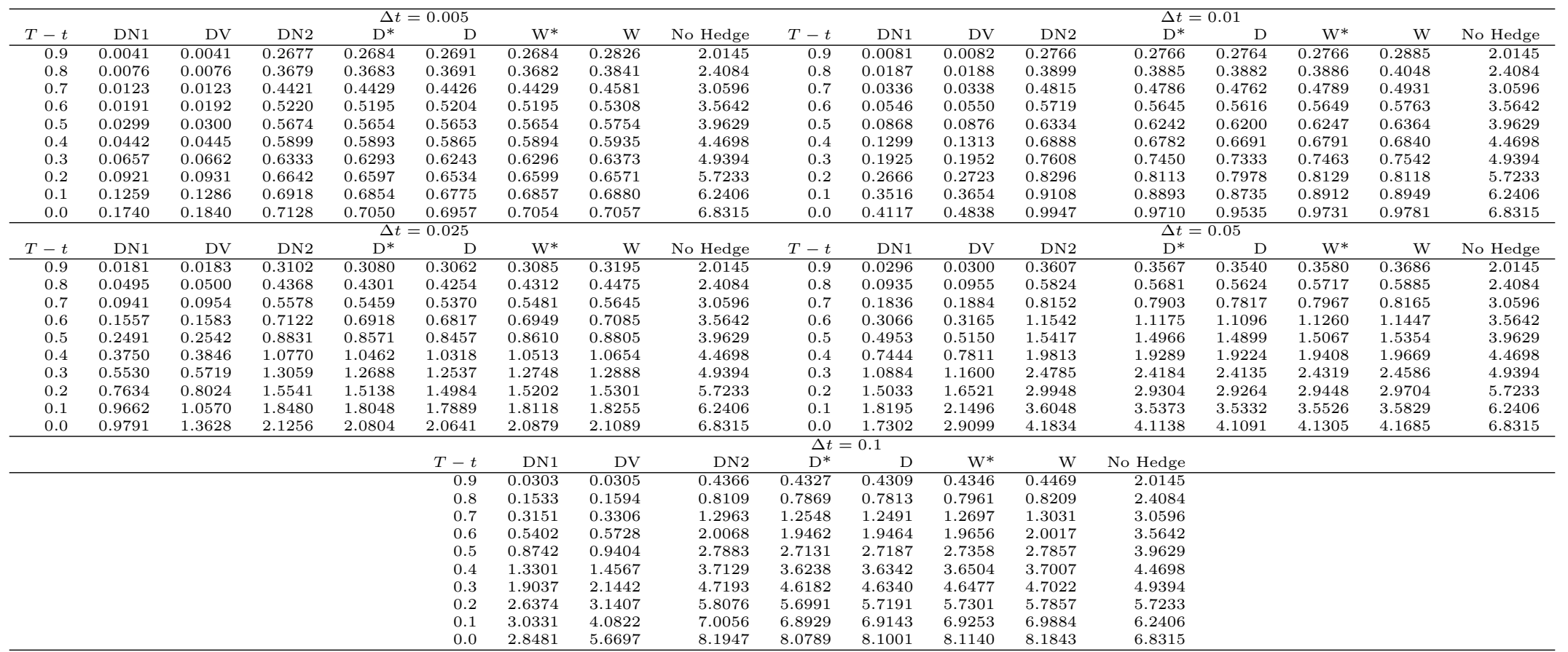

Table 2: Portfolio Standard deviations. DN represents our model's standard deviation. DV represents the Delta/Vega hedging standard deviation. W represents the Wilmott standard deviation, while D represents the Delta hedging standard deviation. Results with an asterisk use greeks calculated from Black-Scholes, rather than the Heston model. 


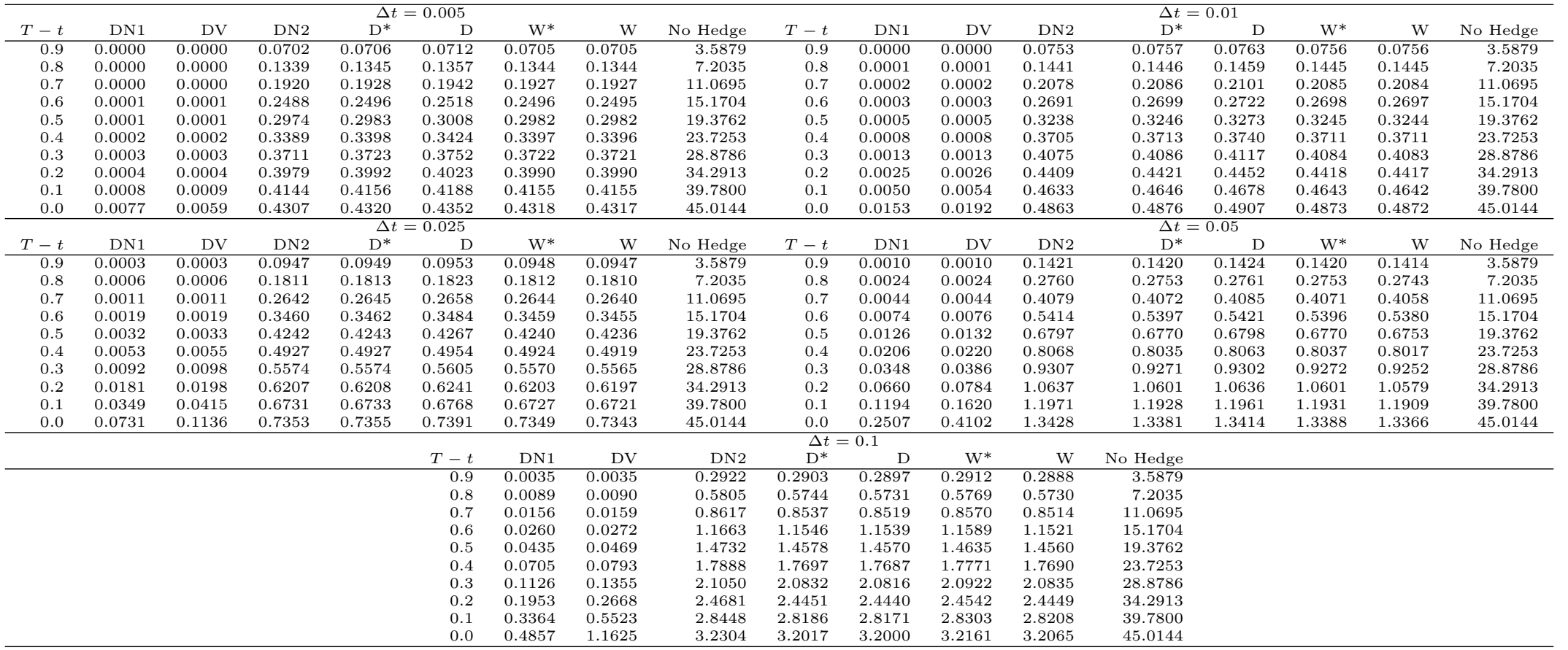

Table 3: Tracking errors. DN represents our model's cumulative tracking error. DV represents the Delta/Vega hedging cumulative tracking error. W represents the Wilmott cumulative tracking error, while D represents the Delta hedging cumulative tracking error. Results with an asterisk use greeks calculated from Black-Scholes, rather than the Heston model. 


\section{Conclusion}

This paper presents a closed form solution for the optimal discrete time hedging strategy for a derivative whose underlying security is subject to stochastic volatility. A number of simpler cases are nested within our model. Simulation results show economically significant improvement from hedging with our model compared to several benchmarks. The relative improvement due to adjusting for discrete hedging is far more substantial when applied to delta/vega hedging than when applied purely to delta hedging as in Wilmott (1994). We conclude that our correction presents a significant improvement to hedging performance at weekly or longer rebalancing intervals. According to the tracking error metric, our model would allow an investor to rebalance his/her portfolio at a monthly interval and achieve comparable performance to using a delta hedge rebalanced every other day.

\section{References}

Bakshi, Gurdip, Charles Cao, and Zhiwu Chen, 1997, Empirical Performance of Alternative Option Pricing Models, Journal of Finance 52, 2003-2049.

Branger, Nicole, and Christian Schlag, 2004, Can Tests Based on Option Hedging Errors Correctly Identify Volatility Risk Premia?, Working Paper.

Dumas, Bernard, Jeff Fleming, and Robert E. Whaley, 1998, Implied Volatility Functions: Empirical Tests, Journal of Finance 53, 2059-2106.

Heston, Steven L., 1993, A Closed-Form Solution for Options with Stochastic Volatility with Applications to Bond and Currency Options, Review of Financial Studies 6, 327-343.

Hutchinson, James M., Andrew W. Lo, and Tomaso Poggio, 1994, A Nonparametric Approach to Pricing and Hedging Derivative Securities Via Learning Networks, Journal of Finance 49, 851-889.

Wilmott, Paul, 1994, Discrete Charms, Risk 7, 48-51.

\section{A Proof of Proposition 1}

Noting that

$$
\begin{aligned}
\Delta f_{1}= & f_{1 s} \Delta S+f_{1 v} \Delta v+f_{1 t} \Delta t+\frac{1}{2}(\Delta t)^{2} f_{1 t t}+\frac{1}{2}(\Delta s)^{2} f_{1 s s} \\
& +\Delta s \Delta v f_{1 s v}+\frac{1}{2}(\Delta v)^{2}+\Delta s \Delta t f_{1 s t}+\Delta v \Delta t f_{1 v t}+\frac{1}{2}(\Delta s)^{2} \Delta t f_{1 s t} \\
& +\frac{1}{2}(\Delta v)^{2} \Delta t f_{1 v v t}+\Delta s \Delta v \Delta t f_{1 s v t}+\frac{1}{6}(\Delta s)^{3} f_{1 s s s}+\frac{1}{2}(\Delta s)^{2} \Delta v f_{1 s s v} \\
& +\frac{1}{2} \Delta s(\Delta v)^{2} f_{1 s v v}+\frac{1}{6}(\Delta v)^{3} f_{1 v v v}+\frac{1}{24}(\Delta s)^{4} f_{1 s s s s}+\frac{1}{6}(\Delta s)^{3} \Delta v f_{1 s s s v} \\
& +\frac{1}{4}(\Delta s)^{2}(\Delta v)^{2} f_{1 s s v v}+\frac{1}{6} \Delta s(\Delta v)^{3} f_{1 s v v v}+\frac{1}{24}(\Delta v)^{4} f_{1 v v v v}
\end{aligned}
$$


with an analogous definition for $\Delta f_{2}$. We then use the Milstein correction to create first order accurate approximations to the evolution of $\Delta v$ and $\Delta s$, we write

$$
\begin{aligned}
& \Delta s=\mu s \Delta t+\sqrt{v} s \Delta z+\frac{1}{2} v s I_{s} \\
& \Delta v=(\alpha-\beta v) \Delta t+\sqrt{v} \sigma_{v} \Delta z_{2}+\frac{1}{4} \sigma_{v}^{2} I_{v} .
\end{aligned}
$$

Here, $\mu, \alpha, \beta$ and $\sigma_{v}$ are all constants, and $d z$ and $d z_{2}$ are correlated Brownian motions with correlation coefficient $\rho$ (a constant). Finally, the Milstein correction is given by

$$
\begin{aligned}
& I_{s}=(\Delta z)^{2}-\Delta t \\
& I_{v}=\left(\Delta z_{2}\right)^{2}-\Delta t
\end{aligned}
$$


The variance of the portfolio's value is then given by:

$$
\begin{aligned}
& F_{1} \equiv E\left((\Delta W)^{2}\right)-(E(\Delta W))^{2} \\
& =\left(v s^{2} \Delta t+\frac{s^{2}}{2} v^{2}(\Delta t)^{2}\right)\left[\pi_{1} f_{1 s}+\pi_{2} f_{2 s}+\pi_{0}\right]^{2} \\
& +2\left(\sigma_{v} \rho v s \Delta t+\frac{1}{4} \rho^{2} \sigma_{v}^{2} v s(\Delta t)^{2}\right)\left[\pi_{1} f_{1 s}+\pi_{2} f_{2 s}+\pi_{0}\right]\left[\pi_{1} f_{1 v}+\pi_{2} f_{2 v}\right] \\
& +\left(2 \mu s^{3} v(\Delta t)^{2}+3 v^{2} s^{3}(\Delta t)^{2}\right)\left[\pi_{1} f_{1 s s}+\pi_{2} f_{2 s s}\right]\left[\pi_{1} f_{1 s}+\pi_{2} f_{2 s}+\pi_{0}\right] \\
& +2\left((\alpha-\beta v) v s^{2}(\Delta t)^{2}+\mu \rho \sigma_{v} s^{2} v(\Delta t)^{2}+2 \sigma_{v} v^{2} s^{2} \rho(\Delta t)^{2}+\frac{1}{2} \rho^{2} v s^{2} \sigma_{v}^{2}(\Delta t)^{2}\right) \\
& {\left[\pi_{1} f_{1 s}+\pi_{2} f_{2 s}+\pi_{0}\right]\left[\pi_{1} f_{1 s v}+\pi_{2} f_{2 s v}\right]} \\
& +\left(2 \rho \sigma_{v}(\alpha-\beta v) v s(\Delta t)^{2}+\rho \sigma_{v}^{3} v s(\Delta t)^{2}+\rho^{2} \sigma_{v}^{2} v^{2} s(\Delta t)^{2}\right) \\
& {\left[\pi_{1} f_{1 s}+\pi_{2} f_{2 s}+\pi_{0}\right]\left[\pi_{1} f_{1 v v}+\pi_{2} f_{2 v v}\right]} \\
& +2\left(v s^{2}(\Delta t)^{2}\right)\left[\pi_{1} f_{1 s}+\pi_{2} f_{2 s}+\pi_{0}\right]\left[\pi_{1} f_{1 s t}+\pi_{2} f_{2 s t}\right] \\
& +2\left(\rho \sigma_{v} v s(\Delta t)^{2}\right)\left[\pi_{1} f_{1 s}+\pi_{2} f_{2 s}+\pi_{0}\right]\left[\pi_{1} f_{1 v t}+\pi_{2} f_{2 v t}\right] \\
& +\left(v^{2} s^{4}(\Delta t)^{2}\right)\left[\pi_{1} f_{1 s}+\pi_{2} f_{2 s}+\pi_{0}\right]\left[\pi_{1} f_{1 s s s}+\pi_{2} f_{2 s s s}\right] \\
& +\left(3 v^{2} s^{3} \sigma_{v} \rho(\Delta t)^{2}\right)\left[\pi_{1} f_{1 s}+\pi_{2} f_{2 s}+\pi_{0}\right]\left[\pi_{1} f_{1 s s v}+\pi_{2} f_{2 s s v}\right] \\
& \left(v^{2} s^{2} \sigma_{v}^{2}\left(1+2 \rho^{2}\right)(\Delta t)^{2}\right)\left[\pi_{1} f_{1 s}+\pi_{2} f_{2 s}+\pi_{0}\right]\left[\pi_{1} f_{1 s v v}+\pi_{2} f_{2 s v v}\right] \\
& +\left(v^{2} s \sigma_{v}^{3} \rho(\Delta t)^{2}\right)\left[\pi_{1} f_{1 s}+\pi_{2} f_{2 s}+\pi_{0}\right]\left[\pi_{1} f_{1 v v v}+\pi_{2} f_{2 v v v}\right] \\
& +2\left(v^{2} s^{3} \sigma_{v} \rho(\Delta t)^{2}\right)\left[\pi_{1} f_{1 s s}+\pi_{2} f_{2 s s}\right]\left[\pi_{1} f_{1 s v}+\pi_{2} f_{2 s v}\right] \\
& +v^{2} s^{2} \sigma_{v}^{2} \rho^{2}(\Delta t)^{2}\left[\pi_{1} f_{1 s s}+\pi_{2} f_{2 s s}\right]\left[\pi_{1} f_{1 v v}+\pi_{2} f_{2 v v}\right] \\
& +\frac{1}{2} v^{2} s^{4}(\Delta t)^{2}\left[\pi_{1} f_{1 s s}+\pi_{2} f_{2 s s}\right]^{2} \\
& +\left(v \sigma_{v}^{2} \Delta t+\frac{1}{8} \sigma_{v}^{4} v^{2}(\Delta t)^{2}\right)\left[\pi_{1} f_{1 v}+\pi_{2} f_{2 v}\right]^{2} \\
& +\left(2 \mu \rho \sigma_{v} s^{2} v(\Delta t)^{2}+2 \sigma_{v} v^{2} s^{2} \rho(\Delta t)^{2}+\frac{1}{2} \rho^{2} v s^{2} \sigma_{v}^{2}(\Delta t)^{2}\right) \\
& {\left[\pi_{1} f_{1 s s}+\pi_{2} f_{2 s s}\right]\left[\pi_{1} f_{1 v}+\pi_{2} f_{2 v}\right]} \\
& +2\left(\mu \sigma_{v}^{2} s v(\Delta t)^{2}+\rho \sigma_{v}(\alpha-\beta v) v s(\Delta t)^{2}+\rho \sigma_{v}^{3} v s(\Delta t)^{2}+\rho^{2} \sigma_{v}^{2} v^{2} s(\Delta t)^{2}\right) \\
& {\left[\pi_{1} f_{1 s v}+\pi_{2} f_{2 s v}\right]\left[\pi_{1} f_{1 v}+\pi_{2} f_{2 v}\right]} \\
& +\left(2(\alpha-\beta v) v \sigma_{v}^{2}(\Delta t)^{2}+\frac{3}{2} v \sigma_{v}^{4}(\Delta t)^{2}\right)\left[\pi_{1} f_{1 v v}+\pi_{2} f_{2 v v}\right]\left[\pi_{1} f_{1 v}+\pi_{2} f_{2 v}\right] \\
& +2 v \sigma_{v}^{2}(\Delta t)^{2}\left[\pi_{1} f_{1 v t}+\pi_{2} f_{2 v t}\right]\left[\pi_{1} f_{1 v}+\pi_{2} f_{2 v}\right] \\
& +2 \sigma_{v} \rho v s(\Delta t)^{2}\left[\pi_{1} f_{1 s t}+\pi_{2} f_{2 s t}\right]\left[\pi_{1} f_{1 v}+\pi_{2} f_{2 v}\right] \\
& +v^{2} s^{3} \sigma_{v} \rho(\Delta t)^{2}\left[\pi_{1} f_{1 s s s}+\pi_{2} f_{2 s s s}\right]\left[\pi_{1} f_{1 v}+\pi_{2} f_{2 v}\right] \\
& +v^{2} s^{2} \sigma_{v}^{2}\left(1+2 \rho^{2}\right)(\Delta t)^{2}\left[\pi_{1} f_{1 s s v}+\pi_{2} f_{2 s s v}\right]\left[\pi_{1} f_{1 v}+\pi_{2} f_{2 v}\right] \\
& +3 v^{2} s \sigma_{v} \rho(\Delta t)^{2}\left[\pi_{1} f_{1 s v v}+\pi_{2} f_{2 s v v}\right]\left[\pi_{1} f_{1 v}+\pi_{2} f_{2 v}\right] \\
& +v^{2} \sigma_{v}^{4}(\Delta t)^{2}\left[\pi_{1} f_{1 v v v}+\pi_{2} f_{2 v v v}\right]\left[\pi_{1} f_{1 v}+\pi_{2} f_{2 v}\right] \\
& +v^{2} s^{2} \sigma_{v}^{2}\left(1+\rho^{2}\right)(\Delta t)^{2}\left[\pi_{1} f_{1 s v}+\pi_{2} f_{2 s v}\right]^{2} \\
& +2 v^{2} s \sigma_{v}^{3} \rho(\Delta t)^{2}\left[\pi_{1} f_{1 s v}+\pi_{2} f_{2 s v}\right]\left[\pi_{1} f_{1 v v}+\pi_{2} f_{2 v v}\right] \\
& \frac{1}{2} v^{2} \sigma_{v}^{4}(\Delta t)^{2}\left[\pi_{1} f_{1 v v}+\pi_{2} f_{2 v v}\right]^{2}
\end{aligned}
$$


which we simplify using the Heston PDE:

$$
(r-q) s f_{1 s}+\frac{1}{2} v s^{2} f_{1 s s}+\frac{1}{2} v \sigma_{v}^{2} f_{1 v v}+\rho v \sigma_{v} s f_{1 s v}+f_{1 t}+(\alpha-\beta v) f_{1 v}=r f_{1}
$$

which in turn implies:

$$
\begin{aligned}
f_{1 s t}= & (q-r) s f_{1 s s}-\rho v \sigma_{v} s f_{1 s s v}-\rho v \sigma_{v} f_{1 s v}-(\alpha-\beta v) f_{1 s v} \\
& -v s f_{1 s s}-\frac{1}{2} v s^{2} f_{1 s s s}-\frac{1}{2} v \sigma_{v}^{2} f_{1 s v v}+q f_{1 s} \\
f_{1 v t}= & (q-r) s f_{1 s v}-\frac{1}{2} s^{2} f_{1 s s}-\frac{1}{2} v s^{2} f_{1 s s v}-\frac{1}{2} \sigma_{v}^{2} f_{1 v v} \\
& -\frac{1}{2} v \sigma_{v}^{2} f_{1 v v v}-\rho \sigma_{v} s f_{1 s v}-\rho v \sigma_{v} s f_{1 s v v}+(\beta+r) f_{1 v}-(\alpha-\beta v) f_{1 v v}
\end{aligned}
$$

Optimal holding of the underlying asset $\left(\pi_{0}\right)$ and a second derivative $\left(\pi_{2}\right)$ is thus determined by the first order conditions for minimising variance of tracking error, which is given by:

$$
\begin{aligned}
F_{1}= & \left(v s^{2} \Delta t+2 q v s^{2}(\Delta t)^{2}+\frac{1}{2} s^{2} v^{2}(\Delta t)^{2}\right)\left(\pi_{1} f_{1 s}+\pi_{2} f_{2 s}+\pi_{0}\right)^{2} \\
& +2\left(\sigma_{v} \rho v s \Delta t+q \sigma_{v} \rho v s(\Delta t)^{2}\right. \\
& \left.+\frac{1}{4} \rho^{2} \sigma_{v}^{2} v s(\Delta t)^{2}+(\beta+r) \sigma_{v} \rho v s(\Delta t)^{2}\right)\left(\pi_{1} f_{1 s}+\pi_{2} f_{2 s}+\pi_{0}\right)\left(\pi_{1} f_{1 v}+\pi_{2} f_{2 v}\right) \\
& +\left(2(\mu+q-r) s^{3} v(\Delta t)^{2}+v^{2} s^{3}(\Delta t)^{2}-\sigma_{v} \rho v s^{3}(\Delta t)^{2}\right)\left(\pi_{1} f_{1 s}+\pi_{2} f_{2 s}+\pi_{0}\right)\left(\pi_{1} f_{1 s s}+\pi_{2} f_{2 s s}\right) \\
& +2\left((\mu+q-r) \rho \sigma_{v} s^{2} v(\Delta t)^{2}+\sigma_{v} v^{2} s^{2} \rho(\Delta t)^{2}\right. \\
& \left.-\frac{1}{2} \rho^{2} v s^{2} \sigma_{v}^{2}(\Delta t)^{2}\right)\left(\pi_{1} f_{1 s}+\pi_{2} f_{2 s}+\pi_{0}\right)\left(\pi_{1} f_{1 s v}+\pi_{2} f_{2 s v}\right) \\
& +\left(\rho^{2} \sigma_{v}^{2} s v^{2}(\Delta t)^{2}\right)\left(\pi_{1} f_{1 s}+\pi_{2} f_{2 s}+\pi_{0}\right)\left(\pi_{1} f_{1 v v}+\pi_{2} f_{2 v v}\right) \\
& +2\left(v^{2} s^{3} \sigma_{v} \rho(\Delta t)^{2}\right)\left(\pi_{1} f_{1 s s}+\pi_{2} f_{2 s s}\right)\left(\pi_{1} f_{1 s v}+\pi_{2} f_{2 s v}\right) \\
& +\left(v^{2} s^{2} \sigma_{v}^{2} \rho^{2}(\Delta t)^{2}\right)\left(\pi_{1} f_{1 s s}+\pi_{2} f_{2 s s}\right)\left(\pi_{1} f_{1 v v}+\pi_{2} f_{2 v v}\right) \\
& +\frac{1}{2} v^{2} s^{4}(\Delta t)^{2}\left(\pi_{1} f_{1 s s}+\pi_{2} f_{2 s s}\right)^{2} \\
& +\left(v \sigma_{v}^{2}(\Delta t)+\frac{1}{8} \sigma_{v}^{4} v^{2}(\Delta t)^{2}+2(\beta+r) v \sigma_{v}^{2}(\Delta t)^{2}\right)\left(\pi_{1} f_{1 v}+\pi_{2} f_{2 v}\right)^{2} \\
& +\left(2(\mu+q-r) \rho \sigma_{v} s^{2} v(\Delta t)^{2}+\frac{1}{2}\left(\rho^{2}-1\right) v s^{2} \sigma_{v}^{2}(\Delta t)^{2}\right)\left(\pi_{1} f_{1 s s}+\pi_{2} f_{2 s s}\right)\left(\pi_{1} f_{1 v}+\pi_{2} f_{2 v}\right) \\
& +\left(2(\mu+q-r) \sigma_{v}^{2} s v(\Delta t)^{2}\right)\left(\pi_{1} f_{1 s v}+\pi_{2} f_{2 s v}\right)\left(\pi_{1} f_{1 v}+\pi_{2} f_{2 v}\right) \\
& +\frac{1}{2} v \sigma_{v}^{4}(\Delta t)^{2}\left(\pi_{1} f_{1 v v}+\pi_{2} f_{2 v v}\right)\left(\pi_{1} f_{1 v}+\pi_{2} f_{2 v}\right) \\
+ & \left(v^{2} s^{2} \sigma_{v}^{2}\left(1+\rho^{2}\right)(\Delta t)^{2}\right)\left(\pi_{1} f_{1 s v}+\pi_{2} f_{2 s v}\right)^{2} \\
+ & \left(2 v^{2} s \sigma_{v}^{3} \rho(\Delta t)^{2}\right)\left(\pi_{1} f_{1 s v}+\pi_{2} f_{2 s v}\right)\left(\pi_{1} f_{1 v v}+\pi_{2} f_{2 v v}\right) \\
+ & \frac{1}{2} v^{2} \sigma_{v}^{4}(\Delta t)^{2}\left(\pi_{1} f_{1 v v}+\pi_{2} f_{2 v v}\right)^{2}+O\left((\Delta t)^{3}\right) \\
&
\end{aligned}
$$


Solving for the first order conditions, and taking a Taylor series expansion, we find the formulae

$$
\begin{aligned}
& \pi_{0}=-f_{2 s} \pi_{2}-\pi_{1}\left[f_{1 s}+\left(\frac{A_{10} f_{2 v}+A_{11} \rho v f_{1 v}}{4 s \sigma_{v} f_{2 v}^{2}\left(1-\rho^{2}\right)}\right) \Delta t\right] \\
& \pi_{2}=-\frac{\pi_{1}}{f_{2 v}}\left[f_{1 v}+\left(\frac{A_{20} f_{2 v}+2 A_{21} v f_{1 v}}{4 \sigma_{v}^{2} f_{2 v}^{2}\left(1-\rho^{2}\right)}\right) \Delta t\right]
\end{aligned}
$$

where the constants $A_{10}, A_{11}, A_{20}$ and $A_{21}$ are given by:

$$
\begin{aligned}
A_{10}= & s^{2} \sigma_{v}\left(f_{2 s s} f_{1 v}-f_{2 v} f_{1 s s}\right)\left[4\left(\rho^{2}-1\right)(\mu+q-r)-2 v+\sigma_{v} \rho\left(\rho^{2}+1\right)\right] \\
& +\rho\left[\left(\sigma_{v}^{4}-2 \rho \sigma_{v}^{3} v\right)\left(f_{2 v v} f_{1 v}-f_{1 v v} f_{2 v}\right)+\left(2 \rho s \sigma_{v}^{3}-4 \sigma_{v}^{2} s v\right)\left(f_{2 s v} f_{1 v}-f_{1 s v} f_{2 v}\right)\right. \\
& -2 \sigma_{v}^{4} v f_{2 v v} f_{1 v v}-2 \sigma_{v}^{2} \rho^{2} v s^{2}\left(f_{2 s s} f_{1 v v}+f_{1 s s} f_{2 v v}\right)-2 v s^{4} f_{2 s s} f_{1 s s} \\
& \left.-4 s \sigma_{v}^{3} \rho v\left(f_{2 s v} f_{1 v v}+f_{1 s v} f_{2 v v}\right)-4 \rho v s^{3} \sigma_{v}\left(f_{2 s s} f_{1 s v}+f_{1 s s} f_{2 s v}\right)-4 \sigma_{v}^{2}\left(1+\rho^{2}\right) v s^{2} f_{2 s v} f_{1 s v}\right] \\
A_{11}= & 2 \sigma_{v}^{4} f_{2 v v}^{2}+4 \sigma_{v}^{2}\left(1+\rho^{2}\right) s^{2} f_{2 s v}^{2}+8 s \sigma_{v}^{3} \rho f_{2 s v} f_{2 v v} \\
& +2 s^{4} f_{2 s s}^{2}+4 \sigma_{v}^{2} \rho^{2} s^{2} f_{2 s s} f_{2 v v}+8 \rho s^{3} \sigma_{v} f_{2 s s} f_{2 s v},
\end{aligned}
$$

and

$$
\begin{aligned}
A_{20}= & \left(f_{2 s v} f_{1 v}-f_{2 v} f_{1 s v}\right) s \sigma_{v}^{2}\left(4\left(\rho^{2}-1\right)(\mu+q-r)+2 \rho^{2}\left(2 v-\rho \sigma_{v}\right)\right) \\
& +\left(\sigma_{v}^{4}-2 \sigma_{v}^{3} \rho^{3} v\right)\left(f_{2 v} f_{1 v v}-f_{1 v} f_{2 v v}\right)+\left(3 s^{2} \sigma_{v}^{2} \rho^{2}-2 \sigma_{v} \rho v s^{2}-s^{2} \sigma_{v}^{2}\right)\left(f_{2 v} f_{1 s s}-f_{1 v} f_{2 s s}\right) \\
& +2 v s^{4} f_{2 s s} f_{1 s s}+2 v \sigma_{v}^{4} f_{2 v v} f_{1 v v}+4 v \sigma_{v}^{2} s^{2}\left(1+\rho^{2}\right) f_{2 s v} f_{1 s v} \\
& +4 v s \sigma_{v}^{3} \rho\left(f_{2 v v} f_{1 s v}+f_{2 s v} f_{1 v v}\right) \\
& +2 v \sigma_{v}^{2} s^{2} \rho^{2}\left(f_{2 s s} f_{1 v v}+f_{1 s s} f_{2 v v}\right)+4 v s^{3} \rho \sigma_{v}\left(f_{2 s s} f_{1 s v}+f_{2 s v} f_{1 s s}\right) \\
A_{21}= & -\left(2 s^{2} \sigma_{v}^{2}\left(1+\rho^{2}\right) f_{2 s v}^{2}+4 s \sigma_{v}^{3} \rho f_{2 s v} f_{2 v v}+s^{4} f_{2 s s}^{2}\right. \\
& \left.+2 s^{2} \sigma_{v}^{2} \rho^{2} f_{2 s s} f_{2 v v}+4 s^{3} \rho \sigma_{v} f_{2 s s} f_{2 s v}+\sigma_{v}^{4} f_{2 v v}^{2}\right),
\end{aligned}
$$

as required. 


\section{B Proof of proposition 2}

The proof follows appendix A except that the variance of the portfolio's value is given by:

$$
\begin{aligned}
F_{2}= & \left(v s^{2} \Delta t+2 q v s^{2}(\Delta t)^{2}+\frac{1}{2} s^{2} v^{2}(\Delta t)^{2}\right)\left(\pi_{1} f_{1 s}+\pi_{0}\right)^{2} \\
& +2\left(\sigma_{v} \rho v s \Delta t+q \sigma_{v} \rho v s(\Delta t)^{2}+\frac{1}{4} \rho^{2} \sigma_{v}^{2} v s(\Delta t)^{2}+(\beta+r) \sigma_{v} \rho v s(\Delta t)^{2}\right)\left(\pi_{1} f_{1 s}+\pi_{0}\right) \pi_{1} f_{1 v} \\
& +\left(2(\mu+q-r) s^{3} v(\Delta t)^{2}+v^{2} s^{3}(\Delta t)^{2}-\sigma_{v} \rho v s^{3}(\Delta t)^{2}\right)\left(\pi_{1} f_{1 s}+\pi_{0}\right) \pi_{1} f_{1 s s} \\
& +2\left((\mu+q-r) \rho \sigma_{v} s^{2} v(\Delta t)^{2}+\sigma_{v} v^{2} s^{2} \rho(\Delta t)^{2}\right. \\
& \left.-\frac{1}{2} \rho^{2} v s^{2} \sigma_{v}^{2}(\Delta t)^{2}\right)\left(\pi_{1} f_{1 s}+\pi_{0}\right) \pi_{1} f_{1 s v}+\left(\rho^{2} \sigma_{v}^{2} s v^{2}(\Delta t)^{2}\right)\left(\pi_{1} f_{1 s}+\pi_{0}\right) \pi_{1} f_{1 v v} \\
& +2\left(v^{2} s^{3} \sigma_{v} \rho(\Delta t)^{2}\right) \pi_{1}^{2} f_{1 s s} f_{1 s v}+\left(v^{2} s^{2} \sigma_{v}^{2} \rho^{2}(\Delta t)^{2}\right) \pi_{1}^{2} f_{1 s s} f_{1 v v}+\frac{1}{2} v^{2} s^{4}(\Delta t)^{2}\left(\pi_{1} f_{1 s s}\right)^{2} \\
& +\left(v \sigma_{v}^{2}(\Delta t)+\frac{1}{8} \sigma_{v}^{4} v^{2}(\Delta t)^{2}+2(\beta+r) v \sigma_{v}^{2}(\Delta t)^{2}\right)\left(\pi_{1} f_{1 v}\right)^{2} \\
& +\left(2(\mu+q-r) \rho \sigma_{v} s^{2} v(\Delta t)^{2}+\frac{1}{2}\left(\rho^{2}-1\right) v s^{2} \sigma_{v}^{2}(\Delta t)^{2}\right) \pi_{1}^{2} f_{1 s s} f_{1 v} \\
& +\left(2(\mu+q-r) \sigma_{v}^{2} s v(\Delta t)^{2}\right) \pi_{1}^{2} f_{1 s v} f_{1 v} \\
& +\frac{1}{2} v \sigma_{v}^{4}(\Delta t)^{2} \pi_{1}^{2} f_{1 v v} f_{1 v}+\left(v^{2} s^{2} \sigma_{v}^{2}\left(1+\rho^{2}\right)(\Delta t)^{2}\right)\left(\pi_{1} f_{1 s v}\right)^{2}+\left(2 v^{2} s \sigma_{v}^{3} \rho(\Delta t)^{2}\right) \pi_{1}^{2} f_{1 s v} f_{1 v v} \\
& +\frac{1}{2} v^{2} \sigma_{v}^{4}(\Delta t)^{2}\left(\pi_{1} f_{1 v v}\right)^{2}+O\left((\Delta t)^{3}\right)
\end{aligned}
$$

which has first order conditions:

$$
\pi_{0}=-\pi_{1}\left(\frac{\sigma_{v} \rho f_{1 v}}{s}+f_{1 s}+\frac{A_{3}}{2 s} \Delta t\right)
$$

where

$$
\begin{aligned}
A_{3}= & -\rho^{2} s \sigma_{v}^{2} f_{1 s v}+\rho^{2} \sigma_{v}^{2} v f_{1 v v}+2 \rho \sigma_{v} s f_{1 s v}(\mu+v-r+q)+2 s^{2} f_{1 s s}\left(\mu+q+\frac{v}{2}-r-\frac{\sigma_{v} \rho}{2}\right) \\
& -2 \sigma_{v} \rho f_{1 v}\left(\beta+q-r+\frac{v}{2}-\frac{\rho \sigma_{v}}{4}\right) .
\end{aligned}
$$

\section{Proof of proposition 3}

The proof follows appendix A except that the variance of the portfolio's value is given by:

$$
\begin{aligned}
F_{3}= & \left(v s^{2} \Delta t+2 q v s^{2}(\Delta t)^{2}+\frac{1}{2} s^{2} v^{2}(\Delta t)^{2}\right)\left(\pi_{1} f_{1 s}+\pi_{2} f_{2 s}+\pi_{0}\right)^{2} \\
& +\left(2(\mu+q-r) s^{3} v(\Delta t)^{2}+v^{2} s^{3}(\Delta t)^{2}-\sigma_{v} \rho v s^{3}(\Delta t)^{2}\right)\left(\pi_{1} f_{1 s}+\pi_{2} f_{2 s}+\pi_{0}\right)\left(\pi_{1} f_{1 s s}+\pi_{2} f_{2 s s}\right) \\
& +\frac{1}{2} v^{2} s^{4}(\Delta t)^{2}\left(\pi_{1} f_{1 s s}+\pi_{2} f_{2 s s}\right)^{2}
\end{aligned}
$$


which has solution

$$
\begin{aligned}
\pi_{2} & =-\pi_{1} \frac{f_{1 s s}}{f_{2 s s}} \\
\pi_{0} & =-\pi_{1} \frac{f_{1 s} f_{2 s s}-f_{1 s s} f_{2 s}}{f_{2 s s}} \\
& =-\pi_{2} f_{2 s}-\pi_{1} f_{1 s}
\end{aligned}
$$

as required. 